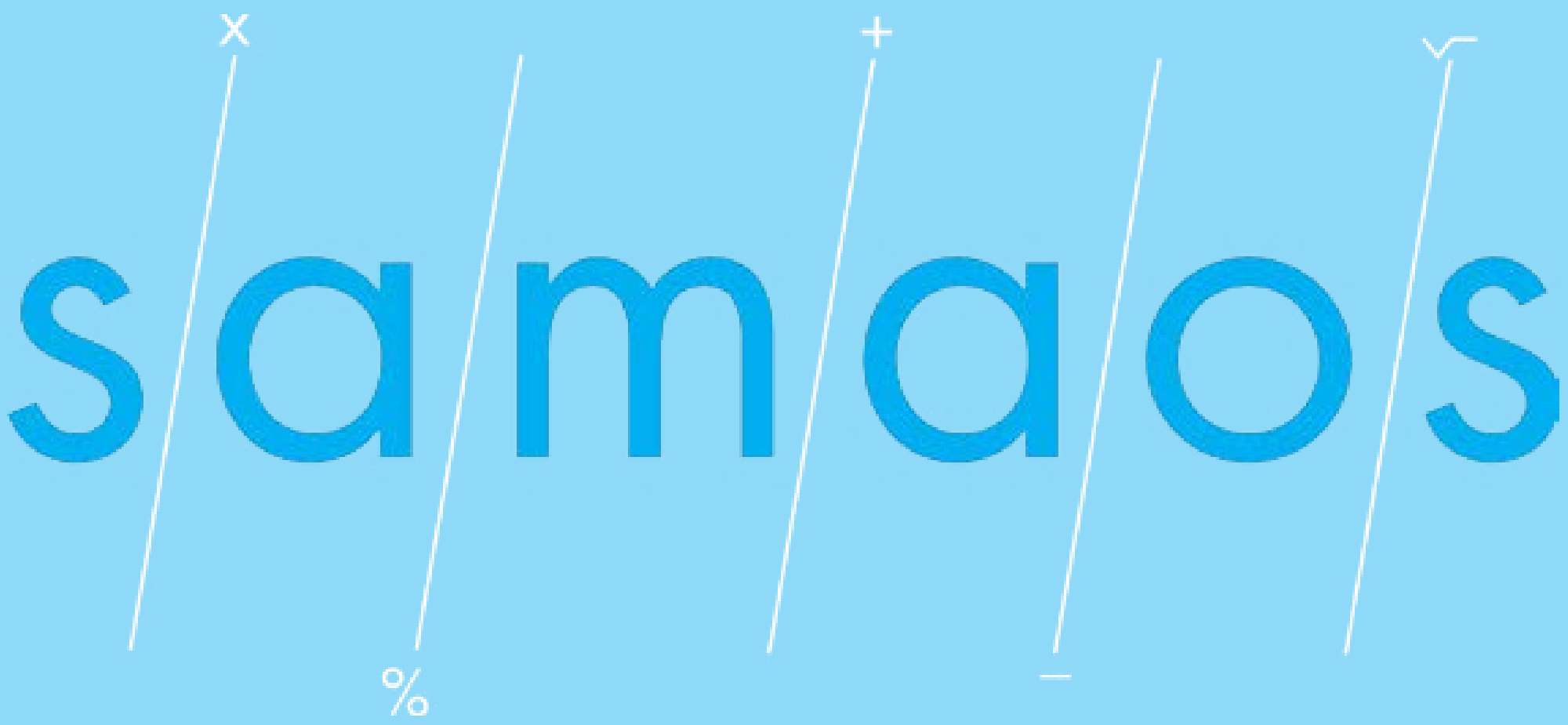

\title{
SAMAOS: Un encuentro para la disertación del saber matemático en la escuela pública colombiana
}

SAMAOS: A meeting for the dissertation of the mathematical knowledge in Colombian public school

SAMAOS: Uma reunião para a dissertação do conhecimento matemático na escola pública colombiana

Sandra Milena Laiton Rozo / Magda Pilar Castellanos Delgado / Oscar Leonardo Cárdenas Forero 


\section{Sandra Milena Laiton Rozo' \\ Magda Pilar Castellanos Delgado² \\ Oscar Leonardo Cárdenas Forero ${ }^{3}$}

1 Colegio Entre Nubes S.O. Magister en Desarrollo Educativo y Social de la Universidad Pedagógica Nacional-convenio CINDE. Correo electrónico: samilaro@hotmail.com y radsanontila@hotmail.com

2 Colegio Entre Nubes S.O. Licenciada en Educación Infantil de la Universidad Pedagógica y aspirante a Magister en Educación de la Universidad Externado de Colombia. Correo electrónico: magdaange12@gmail.com

3 Colegio Entre Nubes S.O. Magister en Desarrollo Educativo y Social de la Universidad Pedagógica Nacional-convenio CINDE. Correo electrónico: osle1972@gmail.com y olcardenasf@ut.edu.co

Fecha de recepción: 23 de febrero de 2015 / Fecha de aprobación: 3 de abril de 2015.

\section{Resumen}

Este artículo de reflexión pretende describir la génesis, intenciones y proyecciones del grupo de maestros "SAMAOS", colectivo pedagógico integrado por docentes de una institución distrital que, desde hace más de un año, viene adelantando exploraciones y reflexiones alrededor del saber matemático y las prácticas de enseñanza que se utilizan para su apropiación. De este modo, se busca mostrar al grupo como un espacio académico y de producción de conocimiento frente a la importancia de la Comprensión Lectora en la resolución de problemas matemáticos en los niños de preescolar y básica primaria.

\section{Palabras clave}

Colectivo de maestros, productor de conocimiento, saber matemático.

\section{Summary}

This reflective article aims to describe the genesis, intentions and projections of the group of teachers "SAMAOS", an educational group integrated by teachers of a public institution, that for more than a year ago has been carrying out explorations and reflections towards the mathematical knowledge and the teaching practices that teachers use for its appropriation. Thus, it seeks to show the group as an academic field and producer of knowledge, towards the importance of Reading Comprehension in Problem Solving in Mathematics in preschool and basic primary.

\section{Key words:}

Group of teachers, producer of knowledge, mathematical knowledge.

\section{Resumo}

Este artigo de reflexão tem como objetivo descrever o gênese, intenções e as projeções do grupo de professores "SAMAOS", composto por um grupo de professores de uma escola pública do distrito, que há mais de um ano, vem realizando explorações e reflexões sobre o conhecimento matemático e práticas de ensino que são utilizados para sua apropriação. Assim, procura-se mostrar o grupo como um campo acadêmico e produtor de conhecimento, que respeita à incidência de compreensão de leitura na resolução de problemas matemáticos no pré-escolar e primário.

Palavras chave:

Grupo de professores, produtor de conhecimento, conhecimento matemático. 


\section{Un poco de historia}

$\mathrm{E}$ grupo que se ha venido consolidando como un equipo pedagógico surge, desde hace año y medio, como producto del interés y la necesidad de reflexionar acerca de la importancia de la comprensión lectora en los procesos de resolución de problemas matemáticos y de los saberes matemáticos en el preescolar y la básica primaria. Este equipo pedagógico, conformado por docentes de preescolar y básica primaria, se gesta a partir de tres puntos fundamentales: el interés investigativo del colectivo, la necesidad de innovar las prácticas pedagógicas y la cualificación y la formación docente.

Todos ellos son aspectos que marcan la diferencia frente a los procesos que se adelantan al interior de la escuela y a los modos como se abordan los diferentes saberes escolares y, en general, los asuntos pedagógicos, epistemológicos, didácticos e innovativos; debido a que permiten validar o invalidar lo que había, lo que hay y lo que se instituye frente al saber mismo, pues constituyen un: "[...] espacio propicio para identificar $\mathrm{y}$ fortalecer las habilidades profesionales y los conocimientos pedagógicos que entran en juego en la especificidad de la actividad docente" (Patiño, Niño, Olmedo, Gaviria, M., Gaviria, A., y Naranjo, 2011, p. 3).

\section{Razón y ser de SAMAOS}

SAMAOS se deriva de la proyección innovativa y pedagógica por explorar los procesos que se desarrollan en torno a las matemáticas, la resolución de problemas y la comprensión lectora de los mismos. Este interés se da solamente para determinar los procesos y habilidades que se usan para la resolución de problemas en el preescolar y la básica primaria, sino también para generar estrategias de apoyo pedagógico y didáctico a aquellos docentes interesados en transformar sus prácticas en esta área, a fin de rescatarla de los procesos mecánicos y técnicos con los que se suele impartir.

Visto esto, el colectivo docente se conjuga como un espacio de encuentro, socialización, puesta de ideas, pensamientos, diálogos y discusiones teóricas, pedagógicas, prácticas, didácticas y epistemológicas, frente a los procesos que orientan el saber matemático en la escuela. Del mismo modo, es un espacio:
[...] natural de desarrollo profesional y de aprendizaje permanente, apropiado para cooperar y compartir [lo pedagógico y lo curricular, y] favorecer la emergencia de nuevos procesos de enseñanza-aprendizaje, así como el desarrollo de conocimientos innovadores en los distintos campos de saber (Patiño, et al. 2011, p. 2).

En ese sentido, el equipo promueve actividades dentro de su haber y saber, como por ejemplo:

- Una revisión a las prácticas de enseñanza de las matemáticas, desde el preescolar hasta la básica primaria, en relación a la comprensión lectora como aspecto que incide en la resolución de los problemas matemáticos.

- El desarrollo de estrategias didácticas de enseñanza-aprendizaje en colectivo, que potencien la transformación de las prácticas pedagógicas al interior del aula.

- La generación de espacios de reflexión pedagógica para la fundamentación y proposición de nuevos escenarios de aprendizaje de la matemática.

- La integración de la investigación y la innovación a los procesos curriculares de la escuela, en tanto permitan la re-significación de los asuntos académicos, pedagógicos y didácticos de la práctica docente.

De esta manera, SAMAOS es una posibilidad para que los docentes se cualifiquen y potencien espacios de formación articulados a nivel institucional, local y distrital, en la medida en que se logren mostrar los hallazgos y las producciones que se plantean al interior del grupo. Puesto que el colectivo: "re-plantea la función "tradicional" del docente como transmisor de conocimientos, hacia la de un "maestro reflexivo", mediador y/o facilitador de aprendizajes" (Patiño, et al. 2011, p. 3) e investigador para la comunidad en general.

En ese sentido, el colectivo, en relación a la cualificación docente, está constituido por un campo de acción con características impresionantes, ya que se asume la investigación:

[...] en el aula como apoyo reflexivo a la práctica profesional [...] [siendo] esencial en la actividad reflexiva de la profesionalidad docente, ya que propicia la actualización de los 
conocimientos y se constituye en el mejor recurso educativo para la adquisición de nuevas metodologías de enseñanza, evaluación y seguimiento de los saberes que se construyen en la actividad del colectivo (Patiño, et al. 2011, p. 7).

Lo anterior, le ha permitido al equipo llevar lo que se ha propuesto a otras instancias y espacios de participación, ya que cuenta con docentes que se desenvuelven en otros escenarios y tienen la posibilidad, y el acceso, de comentar los procesos desarrollados por el colectivo frente al tema que converge al equipo.

Por consiguiente, SAMAOS es una apuesta por el diálogo de saberes, en el que se validan o invalidan las prácticas pedagógicas de los maestros que integran al grupo, dependiendo de si hay relación con lo que se dice y se piensa, o si solamente se hace desde lo que se halla establecido, sin contar con propuestas de estrategias didácticas que permitan a los estudiantes plantear soluciones a los problemas matemáticos con los que se enfrentan diariamente.

En suma, el equipo coadyuva a la construcción de la identidad profesional de los profesionales que lo integran, en la medida en que: "[...] favorece la construcción de significados entre ellos sobre su práctica profesional" (Bolívar, 2006, citado en Huerta, 2011, p. 2); de este modo, adquiere relevancia y pertinencia sobre los procesos y acciones que se instauran al interior de la escuela y los alcances pedagógicos e investigativos que sustenta.

Más aún, en él se posibilita el encuentro con el otro, con su voz, sus ideales, ideas, y contrastes; se enriquece desde lazos afectivos y de respeto que han posibilitado la puesta en escena de inquietudes, intereses, necesidades, metas y objetos de estudio frente a las matemáticas, sus saberes y formas de enseñar. Debido a ello, este proceso de consolidación como colectivo ha tenido sus etapas de encuentro, organización, distribución de tareas, socialización y avances en torno al tema que convoca al grupo.

\section{Dinámicas de trabajo}

Es importante resaltar que cada encuentro del grupo se comprende como un espacio de formación académica, profesional y de cualificación del quehacer pedagógico de los participantes, pero además de reconocimiento del maestro como productor de saber, lo que permite la consolidación misma del equipo, la deliberación, contrastación, aprobación y la proposición pedagógica e investigativa, así como la producción y validación del conocimiento en relación con las prácticas de enseñanza de la matemática y de los discursos que las sustentan.

Este espacio académico, constituido por maestros investigadores interesados en analizar el acontecer de esta disciplina, de producir saber, fortalece la construcción y consolidación de una cultura investigativa, didáctica y científica al interior de la escuela, demostrando con ello que es factible, a pesar de las labores diarias, de las dinámicas escolares y de las responsabilidades continuas, la constitución de colectivos de maestros interesados en la reflexión pedagógica.

Una reflexión que, por medio de la formulación de propuestas de trabajo y de las discusiones y exploraciones que involucra el campo del saber matemático, posibilita la producción de una escuela distinta, en la medida en que se gestan procesos de reflexión y cambio que implican la transformación de los modos como el maestro enseña esta disciplina al interior de las aulas; es decir, las dinámicas del grupo facilitan la transfiguración de las prácticas pedagógicas, pero, además, del maestro mismo, en sus maneras de concebir lo escolar, lo disciplinar, lo pedagógico y lo educativo.

Desde luego, en cada una de las sesiones de encuentro, que se llevan a cabo una vez por semana en los espacios de trabajo pedagógico escolar, en los que se discuten asuntos disciplinares, didácticos y metodológicos, se permite la conjunción de diversas interpretaciones y múltiples miradas, y se asumen unos roles establecidos que ayudan a organizar de un modo particular el accionar del grupo. Aparece un maestro "relator", quien recoge las ideas, planteamientos y, en general, todo lo que acontece en cada reunión, y describe mediante un acta los asuntos que allí se presentan, debaten y reflexionan. Este insumo se lee al comienzo de cada sesión para aprobar su contenido y hacer los ajustes pertinentes, sirve para la producción de escritos posteriores y

para la recolección de la memoria del grupo.

De igual manera, se constituye un maestro "moderador", que se encarga de dar la palabra a los participantes al momento de las intervenciones en cada reunión. Es fundamental reconocer que cada encuentro aborda tres componentes de reflexión: uno epistemológico, que permite conceptualizar las categorías de trabajo planteadas para abordar el objeto de análisis del grupo; otro de carácter didáctico, que posibilita el análisis de los modelos, estrategias y actividades que se proponen hoy para enseñar la 
matemática y, finalmente, uno pedagógico, momento transversal a las discusiones pedagógicas, que facilita hacer cuestionamientos respecto a la escuela, a sus sentidos, a la enseñanza y al maestro en relación con el campo de análisis del grupo.

Unido a esto, cada encuentro se asume desde tres puntos focales; el primero, de presentación y discusión de los documentos asignados para el trabajo, en el que cada participante describe desde su perspectiva los hallazgos, cuestionamientos y logros frente al documento; el segundo, de descripción de los avances y de lectura de las producciones escritas realizadas, en el que se da pie a la confrontación de puntos de vista, a la aclaración conceptual y a la validación de aprendizajes; y un tercer momento, al finalizar las reuniones, en el cual se establecen los acuerdos, se pactan compromisos y se definen las actividades que se llevarán a cabo.

Cabe agregar que el encuentro por lo general cuenta con una agenda de trabajo determinada al finalizar cada sesión, ésta se ajusta al inicio de las mismas dependiendo de la necesidad de involucrar asuntos nuevos y pertinentes, para abordarlos de acuerdo a los propósitos y la ruta de trabajo del colectivo. Es más, cada maestro hace sus búsquedas documentales de forma individual, recurriendo a las fuentes que abordan el campo de análisis y de interés de SAMAOS, pero también lleva a cabo indagaciones que implican lo metodológico, es decir, busca aquel enfoque matemático a adoptar según la pertinencia y relación con los intereses de los participantes.

Esto entre otros asuntos, pues precisamente el espacio de encuentro permite la proposición constante de documentos de lectura de carácter didáctico, epistemológico y, por supuesto, relacionados con el paradigma matemático que orientará el diseño de la estrategia de intervención escolar, así como la producción de matrices de recolección y tablas de análisis de información.

En la actualidad el grupo se encuentra finalizando la fase de exploración, para dar comienzo a la fase de diseño, momento en el que se planeará la propuesta didáctica, para niños y niñas de primaria y preescolar, que pretende el desarrollo de las habilidades de pensamiento que componen la comprensión lectora en matemáticas, base de apoyo a la resolución de problemas matemáticos.

\section{SAMAOS: Reflexiona y propone avances}

Entre las acciones adelantadas por el equipo, se encuentra la socialización de la propuesta ante las directivas institucionales para permitir la realización de aquellas actividades conformes a las intenciones del grupo, debido a ello, dentro de las acciones adelantadas se halla la elaboración de mentefactos, matrices, diseño, ejecución y análisis de encuestas ${ }^{4}$ a docentes de preescolar, primaria y de matemáticas del Colegio Entre Nubes S. O., a fin de percibir si los maestros tienen claras las diferencias de los conceptos: problema, ejercicio, desafío y acertijo, al momento de plantear sus actividades didácticas en el aula.

Dicho trabajo permitió evidenciar que la mayoría de los encuestados visibilizan una diferencia entre problema y ejercicio, mientras que para otros dichos términos son lo mismo, pues sustentan sus apreciaciones bajo las premisas de que ambos términos presentan una pregunta y/o incógnita a resolver, además del uso de unos pasos o métodos a seguir para hallar su respectiva solución ${ }^{5}$.

Del mismo modo, se planteó la conformación de un grupo focal ${ }^{6}$ donde participaron estudiantes que se han destacado por su alto nivel de desempeño académico en el área de matemáticas; quienes se sometieron a una prueba de resolución de problemas por grado en los campos numérico, geométrico, espacial, métrico y aleatorio, con el ánimo de identificar las estrategias, elementos y dificultades que poseen al momento de hacer una lectura del problema, siendo constantemente interrogados por los maestros del grupo respecto a las maneras y acciones adoptadas por ellos para hallar la respuesta y la solución a los mismos.

Paralelamente, dentro del grupo se han ido realizando indagaciones alrededor de varias temáticas, entre ellas: lo que se denomina "comprensión lectora en matemáticas", en sus componentes y en la maneras de desarrollarla en la escuela; exploraciones epistemológicas acerca de los conceptos de problema, ejercicio,

4 Encuesta aplicada en torno a la comprensión lectora en problemas matemáticos, definición, elementos y estrategias empleadas en el aula durante 2014

5 Algunas apreciaciones encontradas fueron: "No."; "No. Ambos requieren de un proceso a seguir"; "No. Ya que en los dos hay que resolver una situación”, entre otras. Perspectiva observada a través de la encuesta a docentes durante 2014.

6 Estudiantes de todos los niveles de preescolar y de básica primaria del Colegio Entre Nubes S.O, de las sedes del colegio y de las dos jornadas. 
desafío y acertijo, con el fin de identificar sus acepciones, sus diferencias y relaciones; se ha abordado también el rastreo del significado de "texto matemático" que describe o enuncia un problema, sus características y maneras de elaborarlos.

De la misma forma, se ha adelantado el estudio de los procesos y habilidades que sustentan la comprensión lectora, que se han materializado en el diseño de mapas conceptuales, mentefactos y matrices analíticas, que recogen información acerca de los sentidos y significados de los conceptos de problema, ejercicio y reto matemático; diferenciación conceptual que se hace necesaria para tener claridad respecto al diseño de una propuesta que favorezca la comprensión lectora en matemáticas, como requisito fundamental para la resolución de problemas por parte de los niños y niñas en preescolar y básica primaria.

Del mismo modo, se han realizado indagaciones respecto a las orientaciones legales locales, distritales, nacionales e internacionales (Estándares Internacionales en Matemática, Lineamientos Curriculares, Estándares Nacionales, Políticas Educativas, etc.) que se han formulado para determinar en ellos la mención a la comprensión lectora como fundamento de la resolución de problemas matemáticos. Complementando lo anterior, se han ido determinado los modos como en las pruebas internacionales (PISA, TIMMS, etc.) se plantean los problemas matemáticos, para determinar su estructura y relaciones con la comprensión lectora.

Estas acciones han venido validando la premisa sobre la importancia de la comprensión lectora en el proceso de resolución de problemas matemáticos por parte de los estudiantes, en tanto que:

[...] la competencia matemática debe incluir la solución de problemas y, de acuerdo a varias metodologías, una acción inicial para solucionarlo es comprender la situación presentada [...] donde la comprensión lectora entra en acción en las sesiones de construcción de conceptos matemáticos, desde una perspectiva constructivista y bajo un enfoque por competencias, la interrelación de las diferentes disciplinas es indispensable y, siendo la lectura un elemento para acceder al conocimiento, es como se concibe el hecho de involucrarla como una herramienta para el aprendizaje de las matemáticas (Allende y Águila, 2012, p. 6).

\section{SAMAOS se proyecta}

El grupo pedagógico pretende trasladar sus reflexiones no solo a la innovación de prácticas de enseñanza de las matemáticas en el aula de preescolar y básica primaria, sino también a nivel institucional, local y distrital, a través de la producción de conocimiento, materializada en la sistematización de experiencias y producciones escritas que permanezcan en la historia de la educación como patrimonio cultural del saber pedagógico.

De esta manera, se intenta enriquecer el trabajo pedagógico en el campo de las matemáticas, cualificando la labor del maestro como deconstructor y constructor de conocimiento, trascendiendo la idea de que es reproductor del mismo. Esto destaca el empoderamiento de su función, en aras de re-significar su misión en la formación de sujetos transformadores, y de posicionar su profesión en la educación colombiana desde la perspectiva de sujetos investigadores que pueden dar cuenta de procesos más enriquecidos, que contribuyan a la disertación colectiva en torno a lo pedagógico, lo académico y lo formativo; todo ello valida: "[...] la visión de todos los seres humanos como sujetos políticos [donde] particularmente, los estudiantes pueden adquirir poder desde la clase de matemáticas" (Sánchez y Duarte, 2009, p. 1).

En suma, SAMAOS le apuesta a la formación de colectivos de maestros cuyo objeto es enriquecer el trabajo pedagógico en el campo matemático, desde la experiencia, el diálogo de saberes y las demandas que exige una comunidad educativa, permeados en la escuela como actores y promotores de la actividad educativa, que enriquecen sus dinámicas de acuerdo a un trabajo en equipo que involucra a los miembros de la comunidad. 


\section{Referencias}

Allende, J., y Águila, M. (2012). La lectura como estrategia de aprendizaje de las Matemáticas. Ponencia presentada en el Congreso Iberoamericano de las Lenguas en la Educación y en la Cultura. IV Congreso Leer.es. Salamanca.

Huerta P., P. (2011). La reflexión de la práctica como elemento de mejora del desempeño académico de los colectivos docentes en educación básica. Ponencia presentada en el XI Congreso Nacional de Investigación Educativa. Obtenido desde http://www.comie. org.mx/ congreso/memoriaelectronica/v11/docs/area_15/0962.pdf

Martínez P., M. (2006). El poder de las experiencias pedagógicas realizadas por colectivos de maestros. ¿Expresiones de acción política? Revista Educación y Ciudad. No. 11. Bogotá: IDEP.
Patiño, S., Niño, M., Olmedo, J., Gaviria, M., Gaviria, S., y Naranjo, M. (2011). El colectivo docente en la Universidad Católica de Pereira. Obtenido desde http://www.ucp. edu.co/ucp_docs/ucp_ colectivo_docente.pdf

Sánchez., y Duarte, J. (2009). Educación matemática crítica: Un abordaje desde la perspectiva sociopolítica a los ambientes de aprendizaje. Ponencia presentada en X Encuentro Colombiano en Educación Matemática. Universidad Distrital Francisco José de Caldas. 
RUTAS POSIBLES EN LA PRODUCCIÓN DE SABER Y CONOCIMIENTO: APUESTAS DE CIUDAD Y REGIÓN 